
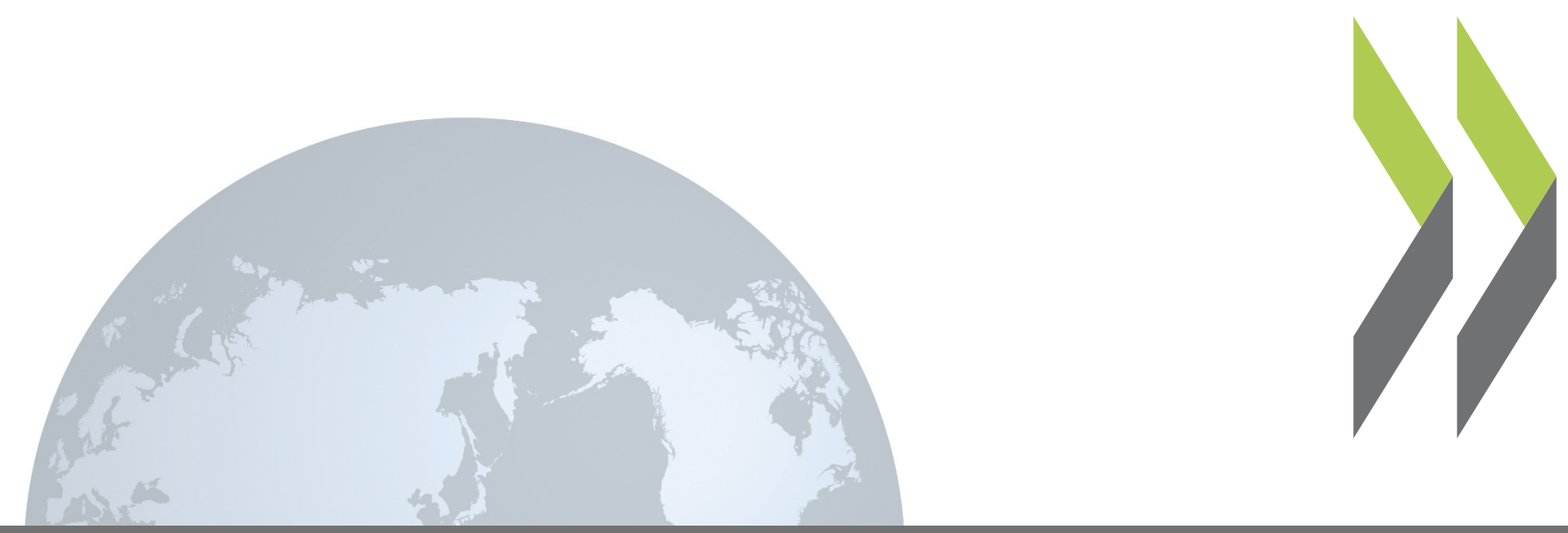

Documents SIGMA No. 15

\title{
Liste de critères de référence
} pour l'élaboration des lois

$$
\begin{aligned}
& \text { et la gestion de la } \\
& \text { réglementation }
\end{aligned}
$$
dans les pays d'Europe centrale et orientale 


\section{LISTE DE CRITÈRES DE RÉFÉRENCE POUR L'É LABORATION DES LOIS ET LA GESTION DE LA RÉ GLE ME NTATION DANS LES PAYS D'E UROPE CE NTRALE ET ORIENTALE}

DOCUMENTS SIGMA : N 15 


\section{LE PROGRAMME SIGMA}

Le Programme SIGMA - Soutien à l'amélioration des institutions publiques et des systèmes de gestion dans les pays d'Europe centrale et orientale - est une initiative conjointe du Centre de l'OCDE pour la coopération avec les économies en transition et du Programme Phare de l'Union européenne. Financée principalement par le Programme Phare, cette initiative vise à aider treize pays en transition à mener à bien la réforme de leur administration publique.

L'Organisation de coopération et de développement économiques est une organisation intergouvernementale qui réunit 29 démocraties à économie de marché avancée. Par l'intermédiaire du Centre, l'OCDE fournit des conseils et une assistance sur les problèmes économiques les plus divers aux pays d'Europe centrale et orientale et aux pays de l'ex-Union soviétique engagés dans des réformes. Le Programme Phare accorde des aides financières à ses partenaires d'Europe centrale et orientale jusqu'à ce qu'ils soient prêts à assumer les obligations liées à la qualité de Membre de l'Union européenne.

Les Programmes Phare et SIGMA couvrent les mêmes pays: Albanie, Bosnie-Herzégovine, Bulgarie, Estonie, Hongrie, Lettonie, Lituanie, ex-République yougoslave de Macédoine, Pologne, Roumanie, République tchèque, Slovaquie et Slovénie.

Créé en 1992, SIGMA dépend du Service de la gestion publique de l'OCDE, qui fournit aux décideurs des informations et des analyses d'experts sur la gestion publique et facilite les contacts et les échanges d'expérience entre responsables de la gestion du secteur public. SIGMA offre aux pays bénéficiaires l'accès au réseau de responsables expérimentés de l'administration publique, aux informations comparées et aux connaissances techniques du Service de la gestion publique.

SIGMA vise à :

- aider les pays bénéficiaires à rechercher des méthodes de gestion publique propres à améliorer l'efficience de l'administration et à encourager les agents du secteur public à respecter les valeurs démocratiques, l'état de droit et les règles de la déontologie ;

- contribuer à mettre en place dans les pays bénéficiaires des structures au niveau de l'administration centrale pour relever les défis de l'internationalisation et des plans d'intégration à l'Union européenne ;

- soutenir les initiatives de l'Union européenne et des autres donneurs visant à aider les pays bénéficiaires à entreprendre la réforme de leur administration publique et contribuer à la coordination des activités des donneurs.

Dans tous ses travaux, SIGMA s'emploie en priorité à faciliter la coopération entre les gouvernements. Cela passe notamment par un soutien logistique à la formation de réseaux rassemblant des praticiens de l'administration publique en Europe centrale et orientale et entre ces praticiens et leurs homologues dans d'autres démocraties.

Les activités de SIGMA se répartissent entre cinq domaines techniques: réforme administrative et stratégies nationales, gestion de la formulation des politiques, gestion des dépenses, gestion des services publics, et supervision administrative. En outre, le Service d'information de SIGMA diffuse des publications et des documents électroniques sur des thèmes intéressant la gestion publique.

Les idées exprimées dans la présente publication ne représentent ni l'avis officiel de la Commission européenne, ni celui des pays Membres de l'OCDE ou des pays d'Europe centrale et orientale participant au Programme. 


\begin{abstract}
AVANT-PROPOS
Les pays d'Europe centrale et orientale doivent repenser leur législation et l'adapter aux critères de l'Union européenne dans la perspective de leur adhésion. L'élaboration d'une législation de bonne qualité, facile à appliquer, revêt donc une importance majeure.

En publiant cette liste de critères de référence, SIGMA propose une méthodologie pour évaluer le cadre institutionnel, les procédures et les techniques d'élaboration des lois adoptés par les pays d'Europe centrale et orientale et vise à recenser les outils auxquels il peut être utile de recourir lorsque des modifications de la législation sont envisagées. SIGMA appuie sa démarche sur l'expérience et les pratiques des pays Membres de l'OCDE, ainsi que sur celles des pays d'Europe centrale et orientale. Cette liste ne se veut pas un exposé de ce qu'il convient de faire, ni un guide sur la façon de traiter certaines activités particulières. Elle vise davantage à dresser un inventaire détaillé des multiples points à prendre en considération pour produire une législation de qualité. Il va de soi que les choix faits dans ce contexte doivent refléter les spécificités locales.
\end{abstract}

Cette publication repose sur un questionnaire établi dans le cadre d'un projet sur la rédaction des lois et la gestion de la réglementation dans les pays d'Europe centrale et orientale que SIGMA a lancé en 1997. Ce projet a donné lieu a la publication d'un document SIGMA intitulé "La rédaction des lois et la gestion de la réglementation dans les pays d'Europe centrale et orientale" qu'il est possible de se procurer sur simple demande auprès de SIGMA. La liste des critères de référence, établie par le Professeur Keith Patchett de l'université de Pays de Galles pour le compte de SIGMA, contient des questions sur la définition de l'action gouvernementale, la structure organisationnelle, les effectifs, les procédures actuelles d'élaboration de la législation et l'évaluation de leur impact. Afin de mettre à disposition des pays d'Europe centrale et orientale un outil méthodologique pratique, la liste de critères de référence adopte une structure qui s'efforce de refléter du mieux possible la chronologie de l'élaboration des instruments juridiques.

La liste des critères de référence a pour objet d'aider le personnel responsable de l'examen et de l'organisation des procédures d'élaboration de la législation, ainsi que le personnel chargé d'élaborer la législation, à mieux prendre conscience des multiples critères qui concourent à une bonne rédaction des lois. Si elle s'adresse plus particulièrement aux fonctionnaires d'Europe centrale et orientale, elle intéresse un lectorat qui dépasse les limites de cette région parce que la plupart des démocraties sont elles aussi en train d'examiner l'élaboration de leur législation et la gestion de la réglementation. La liste des critères de référence peut en outre se révéler un outil utile pour la communauté des donnateurs.

Pour obtenir un complément d'information ou des renseignements sur les activités de SIGMA dans ce domaine, vous pouvez vous adresser à Anke Freibert ou à Michal Ben-Gera à l'adresse ci-dessous.

Un glossaire des termes utilisés dans la liste des critères de référence figure à l'annexe 1.

\author{
SIGMA OCDE \\ 2, rue André-Pascal \\ 75775 Paris Cedex 16, France \\ Tél. (33.1)45.24.79.00 ou 45.24.13.94 \\ Fax (33.1) 45.24.13.00 \\ e-mail : sigma.contact@oecd.org \\ http://www.oecd.org/puma/sigmaweb
}




\section{SYNTHESE}

L'élaboration d'un cadre réglementaire répondant aux critères de l'Union européenne représente une tâche essentielle pour les pays d'Europe centrale et orientale, non seulement pour promouvoir leur développement économique, mais aussi pour assurer le bien-être de leurs citoyens. A cet effet, il convient de mettre en place une structure institutionnelle solide, de disposer de personnel spécialisé dans l'élaboration des lois, de définir des procédures de coordination et de consultation efficientes et de veiller à ce que l'impact des nouveaux instruments juridiques soit évalué avant leur adoption.

Plusieurs pays bénéficiaires ont demandé à SIGMA de les aider à mener à bien cette tâche extrêmement difficile qui consiste à mettre en place des structures et des procédures d'élaboration des lois efficaces et efficientes. Plusieurs ateliers consacrés à des aspects essentiels de l'élaboration des lois ont été organisés. La liste des critères de référence vise à doter les pays concernés d'un outil d'auto-évaluation supplémentaire.

La liste propose un moyen d'évaluer les structures, les procédures et les techniques concourant à préparer et élaborer la législation et de déterminer des méthodes auxquelles il peut être utile de recourir lorsque des modifications de la législation sont envisagées. La rédaction des lois comprend deux phases : La définition de la politique à suivre et la préparation des textes législatifs pour mettre en oeuvre cette politique. La liste des critères de référence s'intéresse à la deuxième phase.

La liste s'appuie sur l'expérience accumulée dans les pays de l'OCDE et de l'Europe centrale et orientale. Elle tient compte, en particulier, des travaux réalisés par le groupe sur la gestion et la réforme réglementaires du service de la gestion publique (PUMA) de l'OCDE, ainsi que des préoccupations du programme PHARE de l'Union européenne exprimées dans le contexte du rapprochement des législations dans la perspective de l'adhésion des pays d'Europe centrale et orientale.

La liste vise à fournir un outil pratique à tous ceux qui ont pour tâche de définir ou de modifier les procédures de rédaction. Elle ne se limite donc pas aux seules questions relatives à la réglementation, mais porte aussi sur les questions d'organisation et de personnel.

L'organisation des procédures de rédaction, sans oublier l'affectation d'effectifs suffisants à cette tâche, conditionne la production d'instruments juridiques de qualité. En outre, l'expérience montre qu'il est important de procéder à toute une série de vérifications tout au long du processus d'élaboration de la politique et de rédaction des textes.

La liste des critères de référence couvrent les thèmes suivants qui sont traités dans des chapitres distincts :

- Contrôles fondamentaux de l'action gouvernementale

- Structures pour l'élaboration des lois

- Elaboration des lois

- Elaboration de la législation dérivée

- Vérifications spécifiques des projets de loi

- Publication des lois et de la législation dérivée

Les questions abordées dans le chapitre sur les structures pour l'élaboration de la législation ont trait au cadre réglementaire, au personnel chargé d'élaborer les textes de loi, aux instruments destinés à vérifier les projets de loi, à l'utilisation de l'informatique et aux procédures de consultation. Les deux chapitres 
suivants portent sur l'élaboration des lois et de la législation dérivée et mentionne un certain nombre de points à examiner sur les procédures, l'attribution des tâches, le calendrier et le rôle du Parlement dans la préparation de ces deux types de textes juridiques.

Pour s'assurer que les procédures d'élaboration des lois produisent des instruments juridiques clairs, cohérents et applicables, un chapitre est consacré à toute une série de vérifications spécifiques comme la conformité des lois et de leurs modifications avec la Constitution, le système juridique existant et les obligations internationales.

Les vérifications portent aussi sur le rapprochement des législations avec le droit communautaire, la mise en oeuvre, la forme, la clarté, la compréhensibilité et l'applicabilité des textes législatifs.

La mise au point de la proposition d'action gouvernementale pendant le processus d'élaboration du texte peut déboucher sur un dispositif législatif couvrant une gamme de sujets plus large ou prévoyant des mécanismes plus complexes qu'il n'était envisagé initialement. Dans ce cas, un deuxième niveau de vérifications peut se justifier à l'issue de l'élaboration du projet.

Les contrôles concernant les sources et la publication de la législation s'intéressent à des points tels que les endroits où l'on peut trouver les textes législatifs (journal officiel, base de données, par exemple), les critères de publication, les autorités responsables, l'accès des partis intéressées et la budgétisation. 


\section{TABLE DES MATIÈRES}

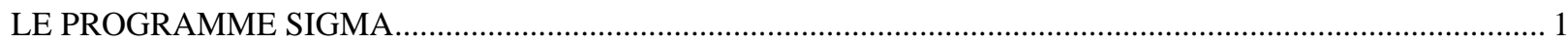

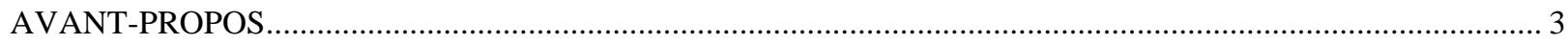

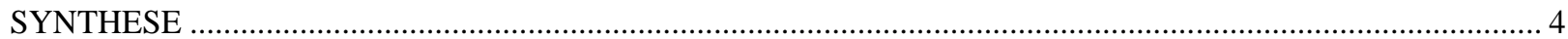

INTRODUCTION

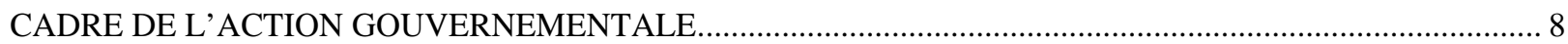

Le texte intégral de la Recommandation figure à l'Annexe 2 à la présente Liste de critères de référence..........8

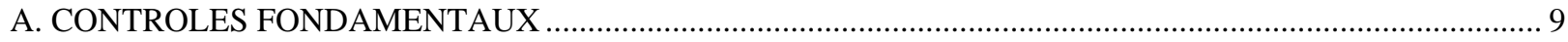

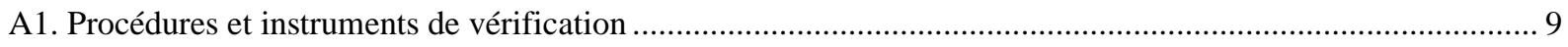

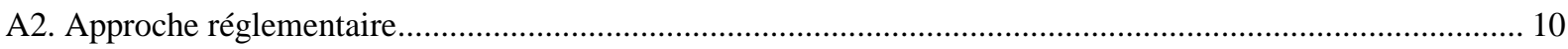

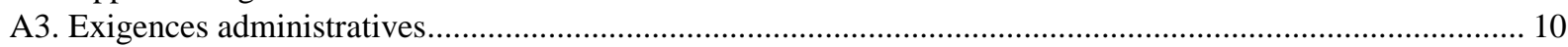

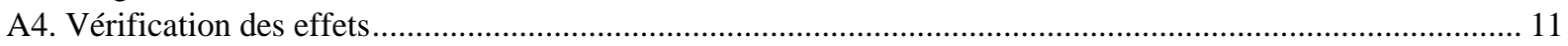

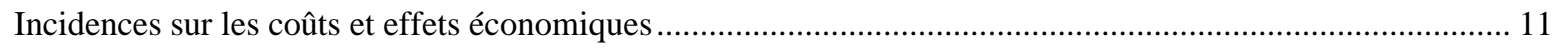

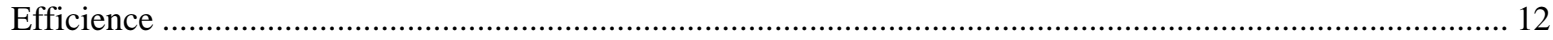

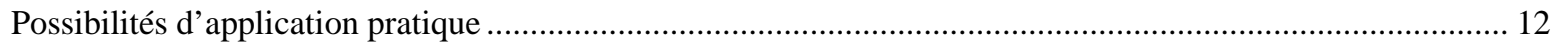

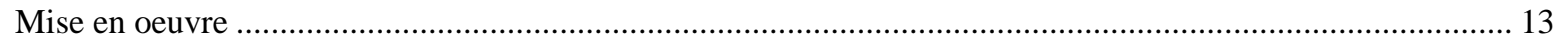

B. STRUCTURES POUR L'ELABORATION DE LA LEGISLATION ..................................................... 13

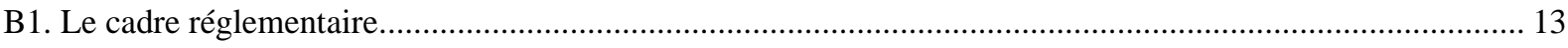

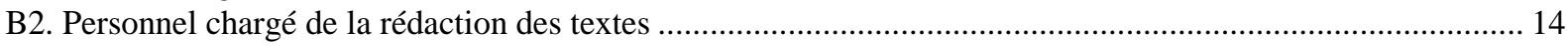

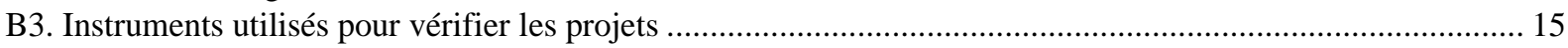

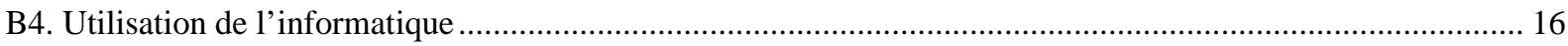

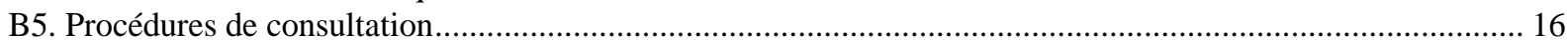

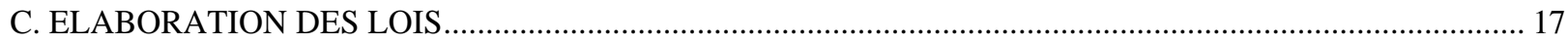

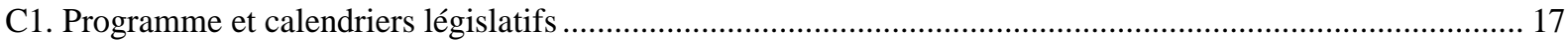

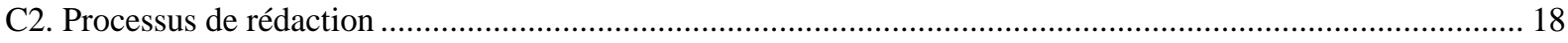

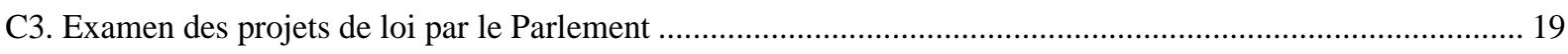

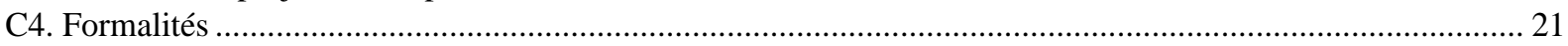

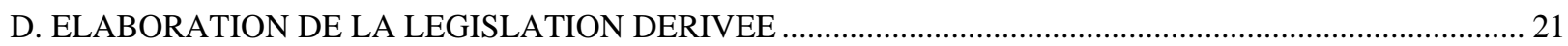

D1. Octroi du pouvoir d'édicter la législation dérivée .......................................................................... 21

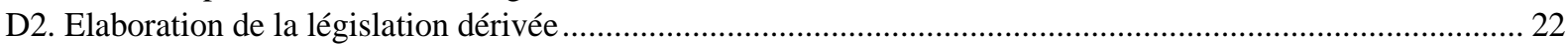

D3. Procédures consécutives à l'élaboration la législation dérivée ............................................................23

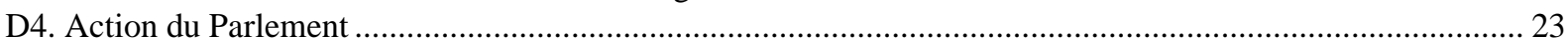

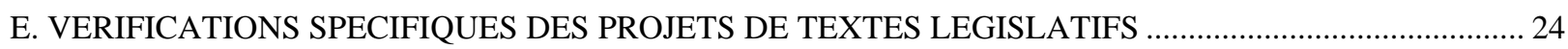

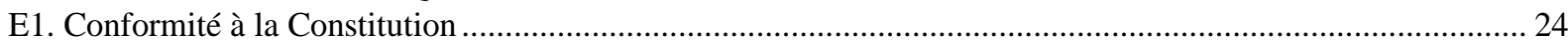

E2. Conformité à la législation et au système juridique existants ........................................................... 24

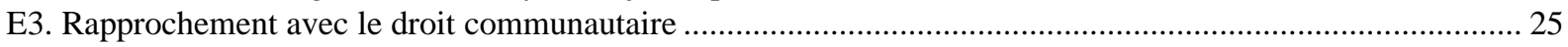

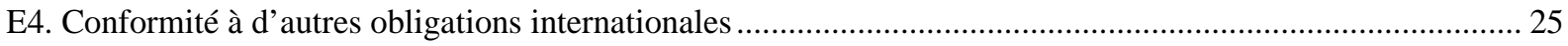

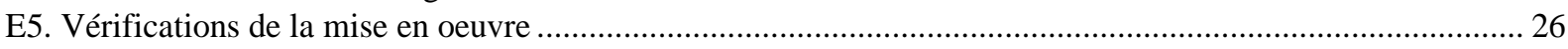

E6. Vérifications concernant la forme juridique, la clarté et la compréhensibilité ..............................................2 27

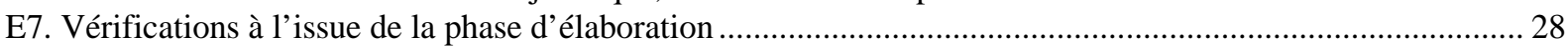

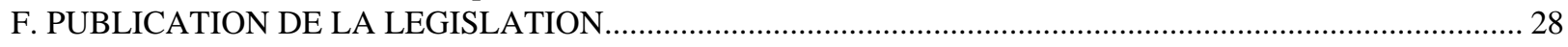

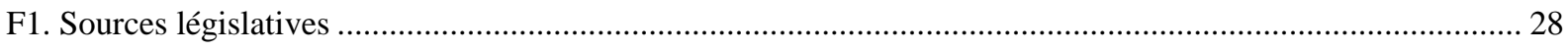

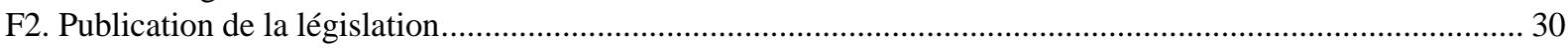

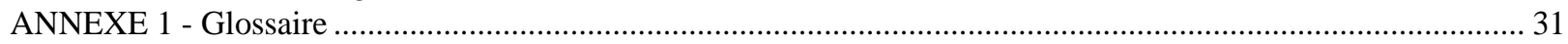

ANNEXE 2 - Liste de critères de référence de L'OCDE pour la prise de décisions en matière de réglementation .. 32 


\section{INTRODUCTION}

En principe, l'élaboration de la législation comporte deux phases : la définition de la politique à mener et l'élaboration du texte législatif ou réglementaire visant à donner effet à la politique adoptée. Lors de la première phase, il faut prendre des décisions fondamentales sur des questions comme :

- laquelle des différentes options envisageables convient-il de privilégier ;

- faut-il réaliser cette option par le recours à la législation plutôt qu'à des moyens non législatifs ;

- quelles autorités devraient être chargées de mettre en application la législation ;

- quelle approche fondamentale convient-il d'adopter dans la législation et quels sont les mécanismes juridiques et administratifs nécessaires pour donner effet à cette approche et permettre son application?

Les questions de ce type doivent être tranchées avant qu'un travail effectif puisse être accompli sur le texte législatif. C'est aux responsables de l'élaboration des politiques spécialistes du domaine en cause, notamment ceux qui possèdent des compétences juridiques spécialisées, qu'il appartient de prendre ces décisions.

Au cours de la seconde phase, ces décisions fondamentales concernant la politique à mener doivent être transformées en un texte juridique, même si des contributions spécialisées et juridiques détaillées sur des questions de fond continueront à être nécessaires. Des compétences juridiques différentes et spécialisées sont indispensables pour transformer les exigences de l'action gouvernementale et les exigences administratives en règles juridiques applicables, efficaces et claires. Les rédacteurs expérimentés de textes législatifs et réglementaires savent très bien comment utiliser les concepts et la terminologie juridiques appropriés dans les contextes législatifs et comment donner effet aux conventions en vigueur quant à la structure, à la forme et au style des textes législatifs et réglementaires.

Dans certains systèmes, ces deux phases sont parfois bien distinctes, mais dans la plupart des pays d'Europe centrale et orientale, elles relèvent normalement des mêmes fonctionnaires ou groupes de travail.

Bien que cette liste de critères de référence concerne principalement la seconde des deux phases, la première section décrit succinctement les types de vérifications qui sont utiles lors de la définition des politiques. Dans de nombreux pays, les rédacteurs de textes législatifs sont invités à préparer des documents explicatifs à l'appui de la législation qu'ils ont rédigée ("exposé des motifs"), dans lesquels doivent figurer des informations sur les raisons qui ont conduit à élaborer les mesures en cause. 


\section{CADRE DE L'ACTION GOUVERNEMENTALE}

Les pays Membres de l'OCDE ont constaté que la qualité de leur législation peut être grandement améliorée si certaines questions sont expressément examinées. Le Conseil de l'OCDE a recommandé (Recommandation adoptée le 9 mars 1995) de toujours poser les questions suivantes lorsqu'une action réglementaire est envisagée :

1. Le problème est-il correctement défini ?

2. L'intervention des pouvoirs publics est-elle justifiée ?

3. La réglementation représente-t-elle la meilleure forme d'intervention gouvernementale ?

4. Quel est le fondement juridique de la réglementation?

5. Quel est (quels sont) le(s) niveau(x) d'administration approprié(s) pour agir ?

6. Les avantages des réglementations en justifient-ils les coûts ?

7. La répartition des effets au sein de la société est-elle transparente?

8. La réglementation est-elle claire, cohérente, compréhensible et accessible aux usagers ?

9. Toutes les parties intéressées ont-elles la possibilité de faire connaître leurs vues ?

10. Comment le respect de la réglementation sera-t-il assuré ?

Le texte intégral de la Recommandation figure à l'Annexe 2 à la présente Liste de critères de référence.

Il est évident que la plupart de ces questions sont des questions fondamentales qui doivent être réglées de manière appropriée avant d'élaborer une législation (par exemple 1-6, 10) et qu'il peut être nécessaire de les poser pour chacune des différentes options envisageables afin de déterminer celle qu'il convient de privilégier. En revanche, la question 8 ne peut être examinée que lorsque la législation est rédigée. Dans certains cas, la question peut être posée à l'un ou l'autre stade (par exemple 7 et 9). De plus, une question qui a déjà fait l'objet d'un examen au stade de l'élaboration de la politique, doit parfois être posée à nouveau au stade de la rédaction lorsque le dispositif détaillé prévu dans le texte prend forme.

Les réponses à toutes ces questions, quels que soient la façon et le moment auxquels elles sont obtenues, sont importantes pour le travail de ceux qui sont chargés de rédiger le texte législatif. De fait, si ces réponses ne sont pas apportées au cours de la phase de définition de la politique, les rédacteurs de textes législatifs se trouveront peut-être dans l'obligation de les demander ou de s'efforcer de les obtenir par eux-mêmes au cours de la phase de rédaction. 


\section{A. CONTROLES FONDAMENTAUX}

Dans les pays Membres de l'OCDE, les vérifications suivantes sont effectuées dans le cadre d'une évaluation ex ante des projets législatifs, afin d'améliorer la fiabilité de la législation, son efficacité et son efficience par rapport à son coût. Le cas échéant, elles devraient être effectuées par chaque organisme chargé d'élaborer la législation -- les lois et, quoiqu'à un moindre degré, la législation dérivée. Ils devraient être intégrés dans les processus d'élaboration normaux utilisés au sein de l'administration publique et, pour ce qui est des textes législatifs et des amendements à ces textes dont il a l'initiative, par le Parlement également.

Ces questions ne portent pas sur le moyen qu'il conviendrait d'utiliser de préférence pour procéder à telle ou telle vérification (par exemple, recours à un organisme spécialisé central agissant pour le compte de tous les ministères ou à des unités spécialisées au sein des différents ministères) ou sur le point de savoir si ces vérifications devraient être opérées en une seule fois. Cela dépend nécessairement de la disponibilité et de l'organisation sur le plan local de ressources spécialisées.

\section{A1. Procédures et instruments de vérification}

A1.1 Les réglementations précisent-elles quels sont les personnes ou les organismes chargés d'effectuer les vérifications suivantes, en particulier lorsque celles-ci doivent être réalisées à l'échelon central ou par différents ministères et quand les travaux peuvent être sous-traités, par exemple à des organismes extérieurs ou des consultants ?

A1.2 A-t-on envisagé de créer une entité publique unique qui puisse acquérir les compétences spéciales requises?

A1.3 Les réglementations indiquent-elles à quel stade du processus d'élaboration, ces vérifications doivent être effectuées (par exemple, lorsque l'on examine les différentes options envisageables, ou une fois qu'une option déterminée a été retenue, ou bien lorsque la mise au point du texte du projet de législation est achevée ou à plusieurs de ces stades) ?

A1.4 Les réglementations prescrivent-elles les procédures à suivre, en particulier dans les cas où l'on constate que les projets envisagés soulèvent des problèmes?

A1.5 Les réglementations stipulent-elles que les exposés des motifs soumis au Conseil des Ministres et au Parlement avec les projets de législation doivent comprendre l'indication expresse que ces vérifications ont été effectuées et, en particulier, des informations sur les coûts prévisibles, l'impact financier et les incidences budgétaires ?

A1.6 Des dispositions spécifiques ont-elles été prises pour veiller à ce que des vérifications analogues soient effectuées pour les projets de textes législatifs ou les modifications de ces textes dont l'initiative revient au Parlement?

A1.7 Des instruments particuliers sont-ils utilisés ou en cours d'élaboration pour faciliter l'évaluation ex ante des propositions de législation (par exemple, modèles informatisés, simulations, listes de critères de référence)? 
A1.8 Un ou des organismes publics sont-ils chargés d'effectuer des recherches sur l'expérience d'autres pays concernant l'utilisation de l'évaluation ex ante, de mettre au point des instruments appropriés en vue de leur application locale et de veiller à informer de leur existence ceux qui sont susceptibles de les utiliser?

A1.9 Les pouvoirs publics ont-ils pris des dispositions pour assurer la formation, dans le pays ou à l'étranger, à la réalisation d'évaluations ex ante systématiques?

\section{A2. Approche réglementaire}

A2.1 Des procédures ont-elles été mises en place pour veiller à ce que le problème à résoudre puisse être identifié de façon précise et exacte, sa nature et son ampleur étudiées de manière approfondie, et ses causes pleinement comprises par les responsables de l'élaboration des politiques et à ce que les objectifs de l'action gouvernementale puissent être définis avec précision?

A2.2 Est-il de pratique courante pour les ministères d'entreprendre une analyse initiale et une vérification des différentes options envisageables afin de déterminer comment atteindre les objectifs de l'action gouvernementale?

A2.3 Est-il d'usage d'effectuer une vérification spécifique afin de déterminer si la législation nouvelle est véritablement nécessaire, par exemple parce que la question :

a) est ou peut être traitée dans le cadre d'une loi en vigueur, ou

b) peut être traitée sans recourir à une action législative (par exemple par des directives administratives, telles que contrat ou négociation et accord avec les parties concernées) ?

A2.4 Les propositions d'action gouvernementale et les options qui s'offrent pour la législation nouvelle font-elles l'objet d'une vérification spécifique, durant les phases d'élaboration de la politique, pour s'assurer qu'elles sont conformes aux exigences de la Constitution ?

A2.5 Les propositions d'action gouvernementale et les options qui s'offrent pour la législation nouvelle font-elles l'objet d'une vérification spécifique, au cours des phases d'élaboration de la politique, pour s'assurer qu'elles sont conformes à la législation en vigueur et aux structures juridiques existantes?

\section{A3. Exigences administratives}

A3.1 Est-il de pratique courante de déterminer, pour chaque nouveau projet législatif, le niveau d'administration le plus approprié auquel le nouveau dispositif devrait être mis en vigueur ?

A3.2 Est-il de pratique courante de procéder à une vérification pour chaque nouveau projet législatif afin de s'assurer :

a) que les structures organisationnelles et les procédures administratives nécessaires pour qu'un nouveau dispositif soit pleinement opérationnel existent déjà et seront suffisantes à cette fin, ou 
b) si elles n'existent pas ou sont jugées insuffisantes, que les moyens supplémentaires nécessaires peuvent être fournis pour permettre une mise en oeuvre efficace?

A3.3 Est-il de pratique courante de procéder à une vérification pour chaque nouveau projet législatif afin de s'assurer :

a) que des ressources suffisantes, en particulier les ressources humaines, existent déjà pour rendre le nouveau dispositif pleinement opérationnel, ou

b) si tel n'est pas le cas, que les ressources nécessaires seront fournies?

A3.4 Au cours de cette vérification, porte-t-on l'attention particulière qui s'impose aux possibilités de recourir au cours du processus de mise en oeuvre à une gestion informatisée et à ses modalités ?

A3.5 Dans l'affirmative, est-il de pratique courante de vérifier les effets sur la mise en oeuvre du recours à des systèmes informatiques (par exemple accroissement ou réduction des coûts ou retards probables dans le lancement du dispositif jusqu'à ce que les systèmes informatiques aient été mis en place et les formations correspondantes menées à bien)?

\section{A4. Vérification des effets}

\section{Incidences sur les coûts et effets économiques}

A4.1 Les réglementations imposent-elles le recours à la vérification des coûts pour les nouveaux projets législatifs?

A4.2 Les réglementations stipulent-elles les types de projets législatifs pour lesquels une évaluation des coûts s'impose, et en particulier indiquent-elles les cas dans lesquels il faut y recourir ?

A4.3 Des procédures ont-elles été officiellement instituées en vue d'évaluer les effets sur le budget de l'Etat des nouveaux projets législatifs, en ce qui concerne les dépenses d'équipement et les dépenses de fonctionnement et, en particulier, les frais de personnel et les coûts administratifs ?

A4.4 Des procédures ont-elles été instituées en vue d'évaluer les incidences sur les budgets d'autres entités publiques (comme les collectivités locales ou les provinces) des nouveaux projets législatifs?

A4.5 Les réglementations imposent-elles que les évaluations des coûts comprennent une évaluation :

a) des incidences financières sur les organismes du secteur privé susceptibles d'être concernés par les nouveaux projets législatifs, notamment sous la forme de coûts administratifs et de dépenses en capital plus importants, et

b) des incidences financières indirectes de la nouvelle législation sur le secteur privé, par exemple sur les stratégies en matière d'investissement et réductions éventuelles des impôts versés? 
A4.6 Les réglementations :

a) imposent-elles que les évaluations des coûts comprennent une évaluation des effets du dispositif proposé en termes de coûts sociaux accrus pour l'ensemble de la collectivité ;

b) fournissent-elles des indications quant aux critères à appliquer pour déterminer si cet accroissement sera acceptable, ou, par exemple, débouchera simplement sur des modifications non voulues du comportement du public ;

c) exigent-elles que soit effectuée une évaluation des conséquences probables à court, moyen ou long terme de la nouvelle législation pour l'emploi ?

A4.7 Des procédures ont-elles été instituées afin de permettre aux pouvoirs publics de déterminer au moyen d'une évaluation ex post si les prévisions des coûts étaient réalistes?

\section{Efficience}

A4.8 Les réglementations imposent-elles que des vérifications soient effectuées pour évaluer si un dispositif législatif envisagé est susceptible d'atteindre ses objectifs ?

A4.9 En particulier, cette vérification est-elle destinée à s'assurer :

a) que le dispositif atteindra ses objectifs au coût le plus bas possible, et

b) s'il risque d'y avoir des effets contraires au but recherché ou des conséquences imprévues et inopportunes?

A4.10 Cette vérification comporte-t-elle l'établissement d'un exposé des avantages attendus de la nouvelle législation (comprenant les données quantitatives appropriées), indiquant en particulier les insuffisances de la législation en vigueur et la manière dont le nouveau dispositif contribuera à améliorer la situation ?

\section{Possibilités d'application pratique}

A4.11 Des vérifications doivent-elles impérativement être effectuées pour évaluer si un dispositif législatif envisagé aura effectivement pour effet :

a) de procurer les avantages prévus à ceux qui sont censés en bénéficier, et

b) de répondre aux besoins opérationnels de l'administration qui est chargée de sa mise en oeuvre?

A4.12 Cette vérification comporte-t-elle un examen visant à déterminer si le dispositif conduira l'administration publique à instituer des pratiques bureaucratiques inutiles ou excessives ou à appliquer des procédures administratives inutilement complexes ?

A4.13 Cette vérification comporte-t-elle un examen afin de déterminer si l'application du dispositif imposera des charges excessives au secteur privé sous la forme de procédures inutilement ou 
excessivement complexes (p. ex. pour obtenir une autorisation officielle) ou de mécanismes d'application complexes?

A4.14 Cette vérification comporte-t-elle un examen afin de déterminer si le dispositif imposera aux administrations publiques ou au secteur privé des exigences ne présentant pas un bon rapport coût-efficacité, c'est-à-dire, en termes économiques, des exigences disproportionnées par rapport aux avantages qu'elles procureront?

A4.15 Cette vérification comporte-t-elle une évaluation de l'impact probable du dispositif à la fois sur ceux auxquels il est principalement destiné et indirectement sur les tiers, pour ce qui est des dépenses ou des restrictions supplémentaires ou de la perte d'avantages ou de possibilités personnelles ou économiques?

A4.16 Cette vérification comporte-t-elle un examen visant à déterminer si le dispositif risque de permettre des pratiques de corruption lors de son application (p. ex. paiements illicites à des fonctionnaires pour obtenir des licences)?

A4.17 Cette vérification comporte-t-elle une évaluation des chances d'acceptation du dispositif par le public une fois qu'il sera opérationnel et des réactions probables du public sous la forme de modifications des comportements (aussi bien les modifications attendues que les modifications imprévues)?

Mise en oeuvre

A4.18 Y a-t-il lieu de soumettre à un examen particulier les méthodes au moyen desquelles le respect de la législation sera assuré et de déterminer :

a) les dispositions juridiques qui seront nécessaires pour assurer la mise en oeuvre effective du dispositif législatif, et

b) les ressources humaines, institutionnelles et financières qui seront nécessaires pour donner effet à la méthode de mise en oeuvre retenue ?

A4.19 Faut-il effectuer une vérification spécifique afin d'évaluer si les méthodes proposées pour assurer le respect des dispositions nouvelles, pour mettre en oeuvre le dispositif législatif et pour régler les litiges, seront efficaces, équitables, cohérentes et ouvertes lors de leur application?

A4.20 Faut-il effectuer une vérification spécifique afin de s'assurer que les méthodes proposées pour l'application, la mise en oeuvre et le règlement des litiges ont de bonnes chances de susciter la confiance et la coopération des personnes concernées et n'excèdent pas leur capacité d'application du dispositif?

\section{B. STRUCTURES POUR L'ELABORATION DE LA LEGISLATION}

\section{B1. Le cadre réglementaire}

B1.1 Existe-t-il des réglementations qui stipulent les procédures et les exigences à respecter pour l'élaboration de la législation? Ont-elles fait l'objet d'une révision depuis la transition? 
B1.2 Ces réglementations sont-elles regroupées dans un instrument réglementaire unique (p. ex. législation, directive ou manuel) qui est utilisé par tous les rédacteurs de textes législatifs ?

B1.3 Ces réglementations prescrivent-elles des procédures types à suivre au cours du processus d'élaboration et des normes types quant à la forme, au format, au style rédactionnel et à la mise en oeuvre de la législation?

B1.4 Un organisme public (p. ex. un ministère) est-il chargé de formuler et d'actualiser ces procédures et exigences et de veiller à ce que les normes communes établies par ces procédures et exigences soient respectées par les rédacteurs de textes législatifs dans les différents ministères?

B1.5 Des listes de critères de référence pour la prise de décision en matière de réglementation sont-elles utilisées à ces fins, ou sont-elles en cours d'élaboration?

B1.6 Existe-t-il un organe parlementaire ou judiciaire expressément chargé de vérifier que les normes sont appliquées de manière cohérente lors de l'élaboration de la législation?

B1.7 Existe-t-il un organisme public unique (p. ex. un ministère) expressément chargé du contrôle du cadre réglementaire et/ou de la coordination du programme d'activités d'élaboration de textes entrepris par les ministères?

\section{B2. Personnel chargé de la rédaction des textes}

B2.1 La rédaction de textes législatifs et réglementaires est-elle reconnue comme une compétence juridique spécialisée dont tous les ministères ont besoin périodiquement et qui nécessite des qualités particulières acquises de préférence grâce à une formation systématique et à une expérience effective?

B2.2 Les différents organismes publics qui sont chargés d'élaborer la législation (p. ex. ministères) disposent-ils, ou ont-ils accès à un nombre suffisant de rédacteurs de textes législatifs expérimentés pour couvrir leurs besoins?

B2.3 Le parlement dispose-t-il, ou a-t-il accès à un nombre suffisant de rédacteurs de textes législatifs expérimentés pour couvrir ses besoins d'élaboration de textes législatifs, ou ceux de ses commissions ou de ses membres, pour présenter des projets de textes législatifs ou préparer des amendements aux projets de loi gouvernementaux?

B2.4 Serait-il plus facile aux ministères de se doter des ressources indispensables en matière de rédaction de textes si leurs budgets comportaient des crédits spécifiques pour l'emploi de rédacteurs de textes législatifs?

B2.5 Des mesures sont-elles prises pour veiller à ce que ceux qui sont chargés de la rédaction de textes législatifs disposent du niveau d'expérience et de formation approprié pour mener à bien les tâches particulières qui leur sont confiées?

B2.6 Y aurait-il avantage à reconnaître spécifiquement, dans le cadre des conditions d'emploi de la fonction publique, les compétences et les qualifications spécialisées requises des rédacteurs de 
textes législatifs? En particulier, existe-t-il des possibilités pour les rédacteurs de textes législatifs de bénéficier d'une promotion ou de tout autre avancement à ce titre ?

B2.7 Les compétences en matière de rédaction de textes législatifs des fonctionnaires chargés d'élaborer la législation font-elles l'objet d'une évaluation officielle et périodique (p. ex., évaluation des performances)?

B2.8 Tous ceux qui peuvent être appelés à élaborer des textes législatifs reçoivent-ils une formation de base aux compétences spécialisées requises pour l'élaboration de textes législatifs ?

B2.9 Une formation est-elle proposée périodiquement aux rédacteurs de textes législatifs pour leur permettre d'améliorer leurs compétences?

B2.10 Des dispositions ont-elles été prises pour permettre à ceux qui n'ont encore jamais pratiqué la rédaction de textes législatifs ou qui n'ont qu'une expérience limitée, de travailler en collaboration ou sous la supervision d'agents plus expérimentés dans la rédaction de textes législatifs?

B2.11 Existe-t-il une politique uniforme quant au moment auquel des consultants ou d'autres experts extérieurs à la fonction publique peuvent être employés pour préparer des projets de textes législatifs ? Les avantages que présente le recours à ces personnes plutôt qu'à des rédacteurs appartenant au ministère font-ils l'objet d'une évaluation périodique ?

B2.12 Des mesures sont-elles prises pour veiller à ce que ces consultants ou experts soient des rédacteurs de textes législatifs compétents et connaissent bien les exigences et les normes en vigueur pour l'élaboration de la législation?

B2.13 La qualité du travail des rédacteurs de textes législatifs (agents appartenant à un ministère ou expert extérieur) fait-elle l'objet d'un contrôle par un haut responsable du ministère ? En particulier, les différents projets font-ils expressément l'objet d'une vérification afin de déceler d'éventuelles erreurs avant que le ministère ne les considère comme achevés ?

B2.14 Les rédacteurs de textes législatifs des différents ministères sont-ils à la disposition des commissions parlementaires ou des commissions analogues pour expliquer ou clarifier les textes dont ils se sont occupés ? Ces organismes sont-ils suffisamment bien informés de la possibilité de faire appel à ces rédacteurs?

\section{B3. Instruments utilisés pour vérifier les projets}

B3.1 Des instruments spécifiques sont-ils utilisés ou sont-ils en cours d'élaboration en vue de faciliter l'évaluation des projets de législation (p.ex. listes de critères de référence portant sur le contenu de la législation ou normes de qualité) ?

B3.2 Un organisme public est-il chargé d'effectuer des recherches sur l'expérience d'autres pays concernant l'utilisation systématique des contrôles au stade de l'élaboration des projets de législation et de mettre au point des instruments appropriés en vue de leur utilisation sur le plan local? 
B3.3 En particulier, utilise-t-on ou envisage-t-on d'utiliser des modèles, des algorithmes, des diagrammes ou des analyses fondés sur la méthode du chemin critique lors de l'élaboration de la législation?

B3.4 Existe-t-il des directives officielles concernant l'utilisation de la législation d'autres pays comme modèle pour les responsables de l'élaboration des politiques ou comme précédent législatif pour les rédacteurs de textes législatifs?

\section{B4. Utilisation de l'informatique}

B4.1 Les pouvoirs publics ont-ils pour politique de tirer pleinement parti de l'informatique au cours du processus d'élaboration de la législation?

B4.2 Tous les rédacteurs de textes législatifs disposent-ils d'un ordinateur personnel à leur usage exclusif de façon à pouvoir composer les projets directement sur ordinateur ?

B4.3 Tous les rédacteurs de textes législatifs utilisent-ils le même logiciel de traitement de texte ? S'agit-il d'une application standard ou des mesures ont-elles été prises pour mettre au point une application spécifiquement destinée à la rédaction de textes législatifs ?

B4.4 Existe-t-il une base de données informatisées officielles sur la législation à laquelle les rédacteurs de textes législatifs peuvent avoir accès en ligne afin de rechercher les textes affectés par les modifications proposées ou les précédents ou bien une telle base de données est-elle en cours d'élaboration?

B4.5 En l'absence d'une base de données officielles, les rédacteurs de textes législatifs ont-ils accès en ligne à une base de données sur la législation dans le domaine de compétence de leur ministère?

B4.6 Le service chargé de l'impression des documents officiels utilise-t-il du matériel d'imprimerie informatisé moderne?

B4.7 Est-il possible techniquement pour les rédacteurs de textes législatifs de transmettre aux services chargés de l'impression des documents officiels le texte législatif qu'ils ont rédigé sous une forme (p. ex. disquette ou en ligne) qui permette son impression sans que d'autres travaux de composition soient nécessaires

\section{B5. Procédures de consultation}

B5.1 A-t-on adopté des réglementations qui stipulent :

a) les procédures types selon lesquelles les consultations avec les personnes concernées doivent être menées, les informations qui doivent être communiquées à ces personnes et les conditions et les délais dans lesquels les réponses de ces personnes doivent être communiquées et examinées ;

b) les mesures à prendre pour que ces consultations soient efficaces, équitables et ouvertes ; 
c) les modalités selon lesquelles le respect des procédures de consultation doit être contrôlé et, dans les cas où ces procédures sont obligatoires, assuré ?

B5.2 Des dispositions officielles ont-elles été prises afin de permettre au grand public de formuler un avis sur les projets de loi ou les projets de législation, stipulant en particulier comment attirer l'attention du public sur les projets de loi et comment rechercher, formuler et examiner les avis exprimés par celui-ci?

B5.3 A-t-on recours à des groupes consultatifs, permanents ou temporaires, afin de faciliter l'élaboration de nouveaux textes législatifs sur des sujets déterminés?

B5.4 Des dispositions officielles ont-elles été prises pour la mise en oeuvre de procédures de consultation pour les textes législatifs d'initiative parlementaire?

B5.5 Les réglementations stipulent-elles que les éléments justificatifs présentés au Conseil des Ministres et au Parlement doivent comprendre une liste des organismes qui ont été consultés au cours du processus d'élaboration du texte?

\section{ELABORATION DES LOIS}

\section{C1. Programme et calendriers législatifs}

C1.1 Les pouvoirs publics conviennent-ils (p. ex. dans le cadre du Conseil des Ministres) d'un programme officiel de projets de loi qui doivent être préparés en vue de leur examen au cours des prochaines sessions du Parlement?

C1.2 Le gouvernement a-t-il mis en place une procédure officielle (p. ex. examen par un comité du Conseil des Ministres) pour déterminer, à partir des demandes concurrentes des ministères, les projets législatifs auxquels il convient d'accorder la priorité dans le prochain programme législatif?

C1.3 Le gouvernement stipule-t-il un calendrier annuel pour déterminer les priorités législatives et pour convenir du prochain programme législatif?

C1.4 Le programme législatif établit-il les priorités législatives du gouvernement pour les prochaines sessions parlementaires, priorité dont il ne s'écartera qu'en cas d'urgence?

C1.5 Ce programme couvre-t-il une période suffisamment longue (p. ex. au moins une année) pour permettre aux ministères concernés de planifier efficacement l'élaboration de leur législation?

C1.6 Le gouvernement collectivement (p. ex. par le biais du Conseil des Ministres) approuve-t-il ou confirme-t-il les orientations de chaque nouveau projet législatif avant que le processus de rédaction soit autorisé à commencer?

C1.7 Un calendrier général est-il fixé pour la préparation des projets de loi dans le programme législatif? Son application fait-elle l'objet d'une coordination et son respect d'un suivi à l'échelon central? 
C1.8 Tous les projets de loi élaborés dans un ministère font-ils l'objet d'une approbation officielle par le ministre compétent une fois la rédaction achevée?

C1.9 Les projets de loi une fois que leur rédaction est achevée doivent-ils être soumis au gouvernement et approuvés collectivement par celui-ci (p.ex. par le Conseil des Ministres) avant qu'ils puissent être présentés au Parlement?

C1.10 Est-il procédé expressément à une comparaison (p. ex. par un comité du Conseil des Ministres) entre les orientations telles qu'elles ont été initialement approuvées et le projet législatif définitif, pour s'assurer de leur cohérence avant que le projet soit approuvé par le gouvernement?

C1.11 Les projets de loi soumis au Conseil des Ministres sont-ils accompagnés d'un document explicatif détaillé (p. ex. exposé des motifs) décrivant les objectifs législatifs et les mécanismes employés et indiquant les vérifications qui ont été effectuées (voir sections $\mathrm{A}$ et $\mathrm{E}$ de la présente liste de contrôle) et leurs résultats?

C1.12 La date du dépôt d'un projet de loi au Parlement est-elle décidée collectivement par le gouvernement (p.ex. par le Conseil des Ministres) ?

C1.13 Le gouvernement avise-t-il suffisamment à l'avance le Parlement du dépôt des différents projets de loi afin de permettre au Parlement de programmer efficacement ses activités législatives ?

C1.14 Les personnes chargées de déposer le projet de loi au Parlement pour le compte du gouvernement et de le présenter et de le défendre disposent-elles d'une note d'information détaillée sur les objectifs et le dispositif législatifs?

C1.15 Des dispositions appropriées ont-elles été prises pour permettre aux rédacteurs de textes législatifs ayant à s'occuper d'un projet de loi de fournir les informations indispensables sur l'élaboration du projet à ces personnes avant et pendant son examen par le Parlement ?

\section{C2. Processus de rédaction}

C2.1 Des mécanismes clairement définis ont-ils été mis en place au sein des ministères pour sélectionner et affecter aux tâches correspondantes les rédacteurs de textes législatifs qui sont chargés de rédiger les projets de loi des ministères ?

C2.2 Lors de l'élaboration d'un projet de loi traitant d'une question complexe ou présentant de multiples aspects, est-il d'usage d'associer des fonctionnaires ayant des compétences particulières dans le domaine en cause ainsi que des fonctionnaires spécialisés dans la rédaction de textes législatifs (par exemple en mettant sur pied une "équipe chargée du projet de loi" ou un groupe de travail au sein du ministère compétent ou par d'autres moyens) ?

C2.3 Existe-t-il des directives claires (notamment des instructions quant aux dépenses autorisées) indiquant à quel moment les différents ministères peuvent faire appel à des personnes extérieures au ministère, et notamment extérieures à l'administration publique, dans le cadre des activités d'une équipe chargée d'un projet de loi ? 
C2.4 Un calendrier est-il fixé au sein du ministère pour l'achèvement de chaque projet de loi ? En particulier, est-il tenu compte du temps indispensable pour préparer les différentes versions du texte législatif concerné qui seront probablement nécessaires, les examiner et organiser des consultations à ce sujet?

C2.5 Est-il de pratique courante pour une équipe chargée d'un projet de loi de définir les objectifs, la méthodologie et l'économie de la législation nouvelle, et en particulier de résoudre les problèmes d'application susceptibles de se poser, avant d'entreprendre le travail systématique de rédaction du texte du projet de loi ?

C2.6 Une fois que les objectifs, la méthodologie et l'économie du projet de loi ont été définis, le rédacteur de textes législatifs se voit-il confier la pleine responsabilité d'élaborer le texte du projet de loi, en vue de son examen par l'équipe chargée du projet de loi ou par les responsables du ministère?

C2.7 Existe-t-il des directives claires (notamment des indications quant aux dépenses autorisées) indiquant à quel moment, à quel stade, et selon quelles modalités, l'équipe chargée du projet de loi peut ou doit consulter d'autres ministères sur le contenu de la législation?

C2.8 Existe-t-il des directives claires indiquant à quel moment et auprès de qui on peut ou doit solliciter les avis de groupes d'intérêts ou d'experts extérieurs à l'administration sur les diverses versions d'un projet de loi ? Ces directives imposent-elles de fixer un délai dans lequel les avis demandés doivent être formulés ?

C2.9 En particulier, existe-t-il des directives claires précisant les cas dans lesquels le texte d'un projet de loi doit être considéré comme confidentiel, et les responsables habilités à autoriser les personnes extérieures à l'administration à formuler des avis sur un projet avant sa présentation au Parlement, et dans quelles conditions?

C2.10 Des directives uniformes ont-elles été édictées pour préciser les personnes qui sont chargées d'examiner attentivement le texte des projets de législation afin de déceler les erreurs ayant trait au contenu du texte, à sa présentation ou à la typographie ? Par exemple, la personne à qui incombe le soin de relire les épreuves de toutes les versions du projet de loi, y compris celle adoptée en définitive par le Parlement, est-elle clairement désignée ?

C2.11 Existe-t-il une procédure selon laquelle le Parlement ou les groupes politiques représentés au Parlement peuvent examiner et formuler des observations sur a) les orientations et les mécanismes législatifs sur lesquels est fondé un projet de loi et/ou b) la forme, la structure ou la rédaction du projet de loi proprement dit avant qu'il soit soumis officiellement au Parlement?

\section{C3. $\quad$ Examen des projets de loi par le Parlement}

C3.1 Un temps suffisant est-il laissé aux membres du Parlement pour étudier le contenu d'un projet de loi et consulter les parties intéressées avant le premier examen de ce projet sur le fond par le Parlement ? En particulier, le règlement intérieur du Parlement contient-il des dispositions à cet égard? 
C3.2 Des explications suffisantes (par exemple éléments justificatifs) sont-elles fournies en même temps qu'un projet de loi est soumis au Parlement? Existe-t-il des directives uniformes concernant le contenu de ces informations et la personne chargée de les préparer?

C3.3 Ces éléments justificatifs sont-ils publiés ou communiqués au public (aux fins d'étude du projet de loi ou lorsque la législation est en vigueur)?

C3.4 Les membres du Parlement peuvent-ils bénéficier d'avis et d'une assistance juridique (par exemple de la part de son secrétariat) pour leur permettre d'examiner efficacement un projet de loi soumis au Parlement, en particulier pour ce qui est du contenu actuel et des insuffisances de la loi que le projet de loi vise à réformer ou à modifier ?

C3.5 Le règlement intérieur du Parlement permet-il au parlement de recueillir des éléments d'appréciation auprès de responsables de ministères, d'experts extérieurs ou de membres bien informés du public lors de l'examen d'un projet de loi ?

C3.6 Les membres du Parlement disposent-ils d'une assistance et de conseils en matière de rédaction (par exemple de la part du secrétariat du Parlement) pour les aider à rédiger des amendements ou leurs propres propositions de loi en vue de les soumettre au Parlement?

C3.7 Lorsque des amendements aux projets de loi sont proposés par le Parlement, existe-t-il des mécanismes appropriés pour veiller à ce qu'ils soient rédigés de manière cohérente avec les objectifs et le libellé du projet de loi ?

C3.8 Le règlement intérieur du Parlement précise-t-il les délais dans lesquels les amendements aux projets de loi doivent être notifiés avant leur examen par le Parlement ? Ces délais sont-ils suffisants pour permettre aux pouvoirs publics, ainsi qu'aux membres du Parlement, d'examiner les incidences d'un amendement?

C3.9 Le règlement intérieur accorde-t-il aux pouvoirs publics des possibilités suffisantes d'examiner les projets de loi et les amendements déposés par les membres du Parlement et de faire connaître ses vues officiellement au Parlement?

C3.10 Si le Parlement présente une proposition de loi, existe-t-il des procédures officielles pour veiller à ce que le Parlement, lorsqu'il élabore la proposition de loi, est informé des contraintes, par exemples financières et opérationnelles, pesant sur les pouvoirs publics dont toute action législative doit tenir compte?

C3.11 Existe-t-il des mécanismes permettant de soumettre les propositions de loi ou les amendements d'initiative parlementaire à des vérifications de leurs incidences analogues à celles qui sont appliquées par le gouvernement pour le projet de loi initial ?

C3.12 Est-il de pratique courante pour les responsables du ministère chargé de l'élaboration d'un projet (en particulier, les rédacteurs de textes législatifs) de suivre l'avancement de l'examen de leur projet au Parlement?

C3.13 Si le gouvernement estime qu'un projet de loi, ou un amendement parlementaire, dont le Parlement est saisi, doit être modifié, le règlement intérieur du Parlement permet-il au ministère ayant élaboré le projet en question de présenter ses propres amendements ? 


\section{C4. Formalités}

C4.1 Les projets de loi qui modifient la législation existante indiquent-ils expressément les dispositions précises qui sont modifiées et précisent-ils la nature de ces modifications ? En particulier, les amendements apportés à la législation prennent-ils la forme de modifications spécifiques ou d'ajouts au texte de cette législation ou de suppressions opérées dans ce texte ?

C4.2 Existe-t-il des règles uniformes prescrivant la manière dont il convient de traiter les dispositions législatives qui ont des incidences financières (par exemple pour les dépenses ou la fiscalité)?

C4.3 Existe-t-il des règles juridiques uniformes prescrivant à quel moment la législation entre en vigueur, si cela n'est pas expressément prévu dans le texte lui-même?

C4.4 Existe-t-il des règles juridiques uniformes régissant l'utilisation de décrets aux fins de l'entrée en vigueur de la législation et les modalités selon lesquelles ce pouvoir peut être exercé ?

C4.5 Existe-t-il un principe juridique selon lequel la législation est réputée tomber en désuétude si elle n'est pas mise en application dans un certain délai?

\section{ELABORATION DE LA LEGISLATION DERIVEE}

\section{D1. Octroi du pouvoir d'édicter la législation dérivée}

D1.1 Existe-t-il des objectifs ou des questions spécifiques pour lesquels il y a lieu de recourir ou de ne pas recourir à la législation dérivée pour mettre en oeuvre la loi ? En particulier, les réglementations stipulent-elles que les questions qui sont susceptibles de revêtir de l'importance pour l'action gouvernementale ou de prêter à controverse doivent être traitées dans le cadre de la loi ?

D1.2 Lorsque la législation dérivée est nécessaires pour mettre en oeuvre la loi, est-il de pratique courante de conférer le pouvoir d'édicter cette législation et de préciser l'organisme auquel ce pouvoir est conféré, dans la loi ?

D1.3 La loi fixe-t-elle des limites ou des conditions à l'exercice, par des organismes publics, de leur pouvoir d'édicter la législation dérivée ?

D1.4 Est-il de pratique courante que la loi impose des limites spécifiques à l'exercice du pouvoir d'édicter la législation dérivée (par exemple en limitant son utilisation aux finalités expressément indiquées) ? En particulier, la loi stipule-t-elle généralement les procédures obligatoires à respecter lors de l'élaboration de la législation dérivée (par exemple en ce qui concerne les consultations)?

D1.5 Est-il de pratique courante au cours de l'élaboration de la loi de vérifier que des pouvoirs juridiques suffisants ont été prévus pour permettre d'édicter toute la législation dérivée qui devrait être nécessaire?

D1.6 Des vérifications sont-elles effectuées au cours de l'élaboration de la loi pour s'assurer que la délégation du pouvoir d'édicter la législation dérivée est conforme à la Constitution ? 
D1.7 Lorsque la mise en oeuvre de la loi nécessite l'adoption d'une législation dérivée pour que le dispositif législatif soit efficace, des vérifications sont-elles effectuées afin de s'assurer que la loi n'entrera pas en vigueur avant que toute la législation dérivée nécessaire ait été élaborée ?

\section{D2. Elaboration de la législation dérivée}

D2.1 Les réglementations précisent-elles quelles sont les personnes à qui il incombe de décider qu'une législation secondaire doit être élaborée pour mettre en oeuvre une loi déterminée, de diriger les procédures qui doivent être suivies lorsqu'une législation dérivée est élaborée, et de déterminer éventuellement les cas dans lesquels l'approbation collective du gouvernement est indispensable?

D2.2 Au sein d'un ministère, est-ce que ce sont les mêmes fonctionnaires que ceux qui ont défini la ligne de conduite pour la loi qui sont généralement chargés de déterminer le contenu de la législation dérivée ? Un rédacteur de textes législatifs est-il associé à cette phase ?

D2.3 L'élaboration de la législation dérivée au sein d'un ministère est-elle en général assurée par un rédacteur de textes législatifs ? Des mesures sont-elles prises pour permettre que cette tâche soit effectuée, si possible, par le rédacteur qui a rédigé la loi ?

D2.4 Les réglementations précisent-elles les autres organismes publics (au sein du ministère concerné ou dans d'autres ministères) qui doivent être associés à l'examen de la législation dérivée ?

D2.5 Les réglementations imposent-elles une obligation quelconque s'agissant de la consultation des parties concernées ou de groupes d'intérêt particuliers?

D2.6 Les réglementations précisent-elles le stade du processus d'élaboration de la législation auquel les consultations internes et la consultation du public doivent avoir lieu et comment elles doivent être menées?

D2.7 En particulier, examine-t-on s'il y a lieu de publier la législation dérivée qui présente de l'importance pour le public, avant son adoption officielle, afin de recueillir les commentaires du public dans un délai déterminé ?

D2.8 Les réglementations imposent-elles expressément des obligations que les rédacteurs de textes législatifs doivent respecter lorsqu'ils élaborent la législation dérivée, en particulier en exigeant que des vérifications soient effectuées pour s'assurer que :

a) l'on dispose de pouvoir juridique indispensable pour édicter la législation dérivée requise ;

b) le contenu de la législation dérivée envisagée est compatible avec la loi qu'elle a pour but de faire appliquer ;

c) la législation dérivée n'est pas en contradiction avec d'autres textes législatifs et réglementaires?

D2 .9 Les réglementations définissent-elles les conventions, notamment en ce qui concerne la forme et le style, que les rédacteurs de textes législatifs sont censés respecter lorsqu'ils rédigent la législation dérivée? 
D2.10 Les réglementations stipulent-elles les cas dans lesquels la législation dérivée doit être soumise à l'approbation du Conseil des ministres, accompagnée de documents explicatifs ?

D2.11 Les réglementations précisent-elles l'action officielle par laquelle un projet d'instrument devient un texte en vigueur (par exemple signature d'un ministre suivie par la publication au Journal officiel) ?

\section{D3. Procédures consécutives à l'élaboration la législation dérivée}

D3.1 Un organe est-il chargé (soit collectivement pour le compte du gouvernement, soit au sein des différents ministères) d'effectuer une dernière vérification, une fois que les textes réglementaires sont élaborés, concernant leur validité ou leur forme, et de définir le pouvoir dudit organisme, notamment à l'égard des instruments présentant des défauts?

D3.2 Existe-t-il une obligation légale imposant que toute la législation dérivée soit enregistrée à l'échelon central et stipulant comment le registre doit être tenu, par qui et les éléments précis qui doivent être enregistrés?

D3.3 Un organisme public est-il chargé d'établir et de publier un index officiel ou un autre guide analogue de l'ensemble de la législation dérivée élaborée et en vigueur pour une loi spécifique ?

D3.4 Les réglementations prescrivent-elles le délai dans lequel la législation dérivée doit être publiée après son adoption?

D3.5 Existe-t-il une obligation légale imposant que, sous réserve des exceptions indiquées, la législation dérivée doit d'abord être publiée pour avoir une portée effective ? Dans la négative, des restrictions sont-elles imposées par la loi quant à l'utilisation de la législation dérivée avant sa publication?

\section{D4. Action du Parlement}

D4.1 Les réglementations stipulent-elles que certains textes de la législation dérivée doivent être communiqués ou signalés au Parlement (par exemple, en raison de leur teneur ou de leur nature controversée) ? Le cas échéant, selon quelles modalités et dans quels délais ces communications doivent être effectuées?

D4.2 Existe-t-il des exigences uniformes quant au moment où la législation dérivée doit être confirmée ou peut être rejetée par le Parlement?

D4.3 Le règlement intérieur du Parlement permet-il l'examen ou l'organisation d'un débat sur la législation dérivée à l'initiative du Parlement ?

D4.4 Le Parlement a-t-il le pouvoir de modifier la législation dérivée élaborée par le gouvernement ? Le règlement intérieur prévoit-il les modalités selon lesquelles ces amendements doivent être présentés et examinés et donne-t-il au gouvernement des possibilités de répondre ?

D4.5 Si le Parlement a le pouvoir d'initiative en matière de législation dérivée, les instruments sont-ils établis par des rédacteurs de texte législatifs expérimentés? 
D4.6 Existe-t-il une procédure selon laquelle les instruments d'initiative parlementaire doivent être soumis au ministère concerné pour observations avant leur approbation par le Parlement ? Existe-t-il une procédure pour veiller à ce que le coût et les incidences économiques de la législation soient pleinement pris en considération au cours du processus d'élaboration?

D4.7 Le Parlement a-t-il créé un comité pour suivre la manière dont les pouvoirs en matière d'élaboration la législation dérivée sont exercés ? Le cas échéant, le règlement intérieur confèret-il des pouvoirs effectifs à cet organe ?

\section{E. VERIFICATIONS SPECIFIQUES DES PROJETS DE TEXTES LEGISLATIFS}

Les vérifications suivantes sont effectuées dans les pays Membres de l'OCDE, au cours de l'élaboration de la législation, pour améliorer sa qualité en tant qu'instrument juridique contenant des dispositions claires, cohérentes, compréhensibles et applicables. Elles devraient être appliquées par tous ceux qui sont chargés d'élaborer les textes juridiques -- aussi bien les lois que la législation dérivée. En conséquence, ces vérifications devraient être des éléments normaux du processus d'élaboration dans les administrations comme au Parlement, pour les textes législatifs et les amendements à ces textes émanant de ces institutions.

\section{E1. Conformité à la Constitution}

E1.1 Les projets de textes législatifs (lois et législation dérivée) font-ils l'objet d'une vérification spécifique, au cours des phases d'élaboration, pour s'assurer de leur conformité à la Constitution?

E1.2 En particulier, la conformité à certaines dispositions constitutionnelles (par exemple les dispositions relatives aux droits de l'homme ou aux droits des citoyens) font-elles l'objet d'une vérification particulière?

E1.3 Le Parlement a-t-il institué des mécanismes spécifiques pour vérifier que les projets de textes législatifs ou les amendements proposés par des parlementaires sont conformes à la Constitution?

E1.4 Les réglementations précisent-elles selon quelles modalités et à qui l'information concernant l'exécution de cette vérification et ses résultats doivent être communiqués, ainsi que les mesures qui peuvent être prises lorsque des difficultés sont constatées?

\section{E2. $\quad$ Conformité à la législation et au système juridique existants}

E2.1 Une vérification particulière est-elle effectuée, avant d'entreprendre l'élaboration d'un texte, pour confirmer que la nouvelle législation est nécessaire et que la question ne peut pas être réglée dans le cadre de la législation existante ou par d'autres moyens ne nécessitant pas un recours à la législation?

E2.2 Tous les projets de textes législatifs (lois ou législation dérivée) font-ils l'objet d'une vérification spécifique par les rédacteurs au cours de la phase d'élaboration pour s'assurer de leur compatibilité avec la législation, les structures et les procédures juridiques existantes ? 
E2.3 Des dispositions ont-elles été prises en vue de vérifier que :

a) le projet de texte législatif est conforme aux dispositions juridiques existantes qui prescrivent des normes d'environnement, et

b) la mise en oeuvre de la législation proposée respectera les normes nationales relatives à la protection de l'environnement?

E2.4 Les réglementations stipulent-elles que les rédacteurs des textes législatifs sont tenus d'effectuer des examens spécifiques, une fois que l'élaboration de la législation nouvelle est achevée, pour déterminer, afin de prendre les dispositions législatives correspondantes :

a) les dispositions de la législation existante qui doivent être abrogées ou modifiées et

b) les dispositions transitoires qui doivent être incluses dans le texte, afin d'assurer la continuité juridique entre la législation nouvelle et la législation existante ?

\section{E3. Rapprochement avec le droit communautaire}

E3.1 Une méthodologie spécifique a-t-elle été mise au point pour s'assurer de la compatibilité de la législation existante ou de la législation nouvelle avec le droit communautaire?

E3.2 Les réglementations prescrivent-elles les types de législation (dans la hiérarchie des instruments législatifs) qui doivent faire l'objet de cette vérification et les critères à appliquer lorsque l'on vérifie la conformité au droit communautaire?

E3.3 Les réglementations indiquent-elles clairement l'organisme ou les organismes chargés de filtrer les projets qui doivent être soumis à vérification et d'appliquer les critères à ces projets ?

E3.4 Les réglementations prescrivent-elles la procédure à suivre si la législation est jugée non conforme à certains aspects du droit communautaire ? Désignent-elles l'autorité à qui appartient la décision finale quant aux mesures à prendre pour les projets de législation qui sont incompatibles avec le droit communautaire applicable?

E3.5 Les réglementations stipulent-elles que tous les projets de législation présentés au Conseil des Ministres doivent être accompagnées d'une déclaration attestant que la vérification a été effectuée à cette fin et indiquant le degré auquel la législation est conforme au droit communautaire (par exemple un certificat de conformité) ?

E3.6 Des dispositifs spécifiques ont-ils été institués pour faire en sorte que les projets de textes législatifs ou les amendements proposés par le Parlement fassent l'objet d'une vérification pour s'assurer de leur conformité au droit communautaire ?

\section{E4. Conformité à d'autres obligations internationales}

E4.1 Des dispositifs ont-ils été expressément institués en vue de vérifier que les projets de testes législatifs sont conformes à la Convention européenne des droits de l'homme? 
E4.2 Des dispositifs ont-ils été institués en vue de vérifier que les projets de textes législatifs sont compatibles avec d'autres obligations conventionnelles déjà souscrites ou susceptibles de l'être, qui peuvent avoir des incidences sur le domaine couvert par la législation (par exemple traités relatifs à des questions d'environnement, conventions de l'OIT) ?

E4.3 Les réglementations désignent-elles les personnes ou les organismes chargés d'effectuer cette vérification? Prescrivent-elles les procédures à suivre, en particulier dans les cas où des divergences sont apparues ?

E4.4 Les réglementations stipulent-elles que tous les projets de textes législatifs transmis au Conseil des ministres doivent être accompagnés d'une déclaration indiquant que la vérification a été effectuée et que la législation est conforme aux conventions internationales ?

E4.5 Des dispositifs ont-ils été institués pour veiller à ce que les projets de textes législatifs ou leurs amendements d'initiative parlementaire fassent l'objet d'une vérification pour s'assurer qu'ils sont conformes aux conventions internationales?

\section{E5. Vérifications de la mise en oeuvre}

E5.1 Les rédacteurs de textes législatifs procèdent-ils à une vérification systématique pour s'assurer que le projet contient toutes les dispositions juridiques nécessaires pour permettre la pleine mise en oeuvre du dispositif et son administration efficace ?

E5.2 Une attention particulière est-elle portée aux méthodes par lesquelles le respect de la nouvelle législation sera assuré et à la détermination des dispositions juridiques qui sont indispensables pour assurer la mise en oeuvre effective du dispositif législatif ?

E5.3 Une vérification spécifique doit-elle être effectuée pour déterminer si les méthodes proposées pour assurer le respect de la nouvelle législation, mettre en oeuvre le dispositif législatif et régler les litiges, seront efficaces, équitables, cohérentes et ouvertes dans leur application?

E5.4 Une vérification spécifique doit-elle être effectuée pour s'assurer que les méthodes d'application, de mise en oeuvre et de règlement des litiges sont de nature à susciter la confiance et la coopération des personnes concernées et n'excèdent pas leur capacité d'application du dispositif?

E5.5 Les rédacteurs de textes législatifs sont-ils tenus de vérifier expressément que la formulation utilisée facilite le travail des tribunaux et le règlement des différends relatifs à ces textes ?

E5.6 Une vérification spécifique doit-elle être effectuée pour déterminer les ressources humaines, institutionnelles et financières requises pour mettre en oeuvre le projet et les améliorations qui pourraient être nécessaires à cet égard et la manière dont elles seront réalisées ?

E5.7 Des dispositions ont-elles été prises pour veiller à ce que cette vérification soit également effectuée pour la législation d'initiative parlementaire ou les amendements à cette législation, qu'ils soient d'initiative gouvernementale ou parlementaire ? 
E5.8 Les réglementations stipulent-elles que les éléments justificatifs soumis au Conseil des ministres ou au Parlement doivent comprendre la notification officielle que cette vérification a été effectuée?

\section{E6. Vérifications concernant la forme juridique, la clarté et la compréhensibilité}

E6.1 Les réglementations stipulent-elles les formules normalisées que les rédacteurs de textes législatifs doivent normalement respecter lorsqu'ils élaborent la législation, en particulier en ce qui concerne :

a) la forme des instruments - loi et législation dérivée - (par exemple, intitulé, mode de numérotation des instruments et mode de division et de numérotation des différentes sections internes) ;

b) les formules liminaires et finales et les dispositions techniques traitant des objectifs, du fonctionnement et de l'application du dispositif ;

c) l'endroit où faire figurer certains types particuliers de dispositions législatives (par exemple, les dispositions relatives à la mise en oeuvre, la prolongation ou la modification d'autres législations, les dispositions transitoires);

d) les formules à utiliser pour des types particuliers de dispositions législatives (par exemple, dispositions pénales, dispositions relatives à la modification et à l'abrogation du texte en cause) ;

e) la mise en page et les caractères utilisés pour l'impression ?

E6.2 Les réglementations stipulent-elles des pratiques normalisées à suivre lors de la rédaction du texte législatif (par exemple, structure du texte législatif, style de rédaction, terminologie, définition, cohérence interne et souci d'éviter toute ambiguïté) ?

E6.3 Des dispositions expresses ont-elles été prises pour veiller à ce que les rédacteurs de textes législatifs, aussi bien ceux qui appartiennent à l'administration que ceux qui travaillent pour le Parlement, adoptent les mêmes formules normalisées et suivent les mêmes pratiques en matière de rédaction?

E6.4 Les pouvoirs publics ont-ils institué pour politique que la législation doit :

a) être rédigée de manière à être facile à comprendre et à appliquer ;

b) être claire quant à la manière dont son contenu est énoncé ;

c) éviter les termes juridiques excessivement techniques ;

d) être rédigée dans une langue d'usage courant pour le public visé par la législation ?

E6.5 Des dispositions ont-elles été prises en vue d'effectuer une vérification systématique que toutes les législations nouvelles respectent ces normes?

E6.6 Les rédacteurs de textes législatifs sont-ils encouragés à se mettre d'accord sur des conventions rédactionnelles expressément conçues pour donner effet à ces objectifs ? 
E6.7 Un organisme est-il chargé de déterminer les moyens d'améliorer la qualité de la rédaction des textes législatifs, en particulier la formulation et le style, afin de rendre la législation plus accessible?

\section{E7. Vérifications à l'issue de la phase d'élaboration}

La mise au point de la proposition d'action gouvernementale au cours du processus d'élaboration du texte peut déboucher sur un dispositif législatif qui couvre un ensemble de questions plus large ou qui prévoit des mécanismes plus complexes que ce qui était envisagé initialement. En pareils cas, de nouvelles vérifications à un second niveau peuvent se révéler appropriées une fois que l'élaboration du projet est achevée. Ces vérifications peuvent également être nécessaires pour établir l'exposé des motifs qui accompagne généralement les lois.

En particulier, ces vérifications devraient concerner à nouveau des questions examinées précédemment sous les rubriques suivantes de la liste:

1. Exigences administratives : A3.2-3

2. Coûts et effets économiques : A4.3-5

3. Efficience : A4.9

4. Possibilités d'application pratique : A4.11-16

5. Mise en oeuvre : A4.19-20.

E7.1 Les réglementations stipulent-elles à quel moment des projets de législation dont l'élaboration est achevée (par exemple, des projets susceptibles d'avoir des effets socio-économiques importants) doivent faire l'objet de nouvelles vérifications avant leur adoption par le Conseil des ministres et leur soumission au Parlement?

E7.2 Des procédures ont-elles été mises en place pour renvoyer ces projets à l'organisme chargé d'effectuer les vérifications afin que ses conclusions puissent être prises en compte par le rédacteur du texte lorsqu'il révisera le projet?

\section{F. PUBLICATION DE LA LEGISLATION}

\section{F1. Sources législatives}

F1.1 Existe-t-il un journal officiel dans lequel les textes officiels authentiques de la législation, y compris les projets de loi, sont publiés?

F1.2 Les réglementations exigent-elles que l'ensemble de la législation (lois et législation dérivée) soit publiée dans le journal officiel immédiatement après son adoption?

F1.3 Les réglementations stipulent-elles que l'ensemble de la législation doit être publiée une deuxième fois dans des recueils annuels? 
F1.4 Existe-t-il un registre central ou un système d'enregistrement de l'ensemble de la législation permettant de créer et de tenir des archives exhaustives?

F1.5 Si une base de données informatisée relative à la législation a été mise en place par les pouvoirs publics ou si sa création est envisagée :

a) des mesures ont-elles été prises pour veiller à ce qu'elle contienne la législation sous une forme dont l'Etat peut garantir l'authenticité, et

b) les juristes, les juges et le public pourront-ils y avoir accès en ligne, à l'instar des fonctionnaires?

F1.6 Les réglementations imposent-elles qu'une codification de la loi et de la législation dérivée (contenant l'ensemble des textes en vigueur à la date de publication) soit préparée et publiée périodiquement?

F1.7 Existe-t-il des dispositions permettant de réimprimer toute législation profondément modifiée sous une forme qui est authentique?

F1.8 A-t-il été institué une obligation de publier périodiquement un index officiel à jour de la législation en vigueur qui indique également sur quels points, quand et comment la législation antérieure a été modifiée ?

F1.9 Chaque ministère a-t-il l'obligation de tenir à jour une collection exhaustive de la législation existante dans les domaines relevant de sa compétence?

F1.10 Chaque ministère a-t-il facilement accès à une collection exhaustive de toute la législation en vigueur ? En particulier, tous les rédacteurs de textes législatifs ont-ils généralement accès à un jeu complet de la législation en vigueur?

F1.11 Des recueils exhaustifs de la législation en vigueur sont-ils conservés dans des centres auxquels les membres des professions juridiques et le public ont facilement accès ? Des fonds suffisants sont-ils disponibles pour permettre d'une manière générale la bonne gestion de ces recueils?

F1.12 Les juristes et les entreprises privées peuvent-ils acquérir facilement un jeu authentique et complet de la législation en vigueur ? Des exemplaires des différents instruments sont-ils aisément disponibles dans l'ensemble du pays, à un prix abordable pour le grand public ?

F1.13 Les pouvoirs publics ont-ils confié à un ou à des organismes créés à cet effet, ou existants, le soin de :

a) suivre en permanence l'état de la législation (par exemple, en vue de présenter des propositions d'abrogation des textes qui sont dépassés ou qui ne sont plus efficaces), et

b) préparer et publier périodiquement des codifications des lois et/ou de la législation dérivée en vigueur? 


\section{F2. Publication de la législation}

F2.1 Les réglementations prévoient-elles un délai pour l'impression des projets de loi après leur approbation par le gouvernement ou le dépôt de propositions de loi par les membres du Parlement et avant leur soumission au Parlement?

F2.2 Les réglementations stipulent-elles à quel stade du processus d'élaboration un projet de loi doit être publié, à quel moment il doit être mis à la disposition du public (par exemple, pour que celui-ci ait la possibilité de l'acheter) et à qui les exemplaires doivent être automatiquement fournis gratuitement?

F2.3 Les réglementations indiquent-elles sur quels budgets (budget général de l'Etat, des différents ministères, du Parlement) les coûts d'impression et de publication de la législation doivent être imputés?

F2.4 Les réglementations précisent-elles qui a le pouvoir d'apporter des modifications au texte de la législation après son adoption, mais avant sa publication définitive, et dans quel cas (par exemple, pour corriger les erreurs typographiques)?

F2.5 Les réglementations stipulent-elles dans quels délais la législation doit être officiellement publiée après son adoption et sa promulgation, et les éventuels cas exceptionnels dans lesquels elle peut entrer en vigueur avant sa publication? 


\section{ANNEXE 1 - Glossaire}

Législation modificatrice: législation qui modifie l'effet de la législation existante en modifiant, remplaçant ou abrogeant certaines dispositions ou en en ajoutant de nouvelles.

Rapprochement des législations : processus consistant à harmoniser le contenu de la législation existante avec le droit communautaire.

Projet de loi : projet de texte législatif soumis au Parlement.

Codification : processus consistant à établir un recueil exhaustif et cohérent de la législation, ou des règles législatives, sur un sujet déterminé et dans un format organisé.

Evaluation ex ante : processus consistant à évaluer qualitativement un élément de la législation proposée avant qu'une décision définitive soit prise quant à son adoption.

Rédacteur de textes législatifs : personne (fonctionnaire ou consultant sous contrat) qui a pour activité d'élaborer le texte de la nouvelle législation.

Elaboration de la législation: processus consistant à transformer une nouvelle politique en règles juridiques énoncées dans la législation, sous la forme et dans le style juridique appropriés, préalablement à l'adoption d'un texte législatif.

Législation: droit écrit adopté par le Parlement, le gouvernement ou un autre organe investi (généralement par la Constitution) du pouvoir d'édicter des instruments normatifs.

Loi : ensemble de la législation adoptée par le Parlement (c'est-à-dire l'organe investi de la fonction principale d'élaboration de la loi au sein de l'Etat).

Cadre réglementaire : ensemble des dispositions ordinaires (imposées par la loi) régissant l'élaboration de la législation, notamment les éventuelles obligations légales quant aux procédures normalisées à suivre et quant à la forme et au style selon lesquels la législation doit être rédigée.

Réglementation : tout instrument parmi l'ensemble des instruments juridiques qui peuvent être adoptés par le Parlement, le gouvernement ou l'administration publique pour influer sur les comportements (c'est-à-dire les textes législatifs tels que les lois et la législation dérivée, et les instruments tels que circulaires et instructions).

Gestion de la réglementation: gestion systématique des processus d'élaboration, de rédaction et d'adoption des textes législatifs et réglementaires.

Législation dérivée: instruments normatifs (par exemple, décrets, directives, règlements et réglementations, arrêtés) adoptés par le gouvernement ou un autre organe exécutif investi du pouvoir d'édicter de tels instruments aux fins de l'application des lois.

Vérification : processus consistant à examiner attentivement une proposition d'action gouvernementale ou un projet de législation afin de vérifier que cette proposition ou ce projet est conforme à une norme déterminée ou présente la qualité requise. 


\title{
ANNEXE 2 - LISTE DE CRITÈRES DE RÉFÉRENCE DE l'OCDE POUR LA PRISE DE DÉCISIONS EN MATIÈRE DE RÉGLEMENTATION
}

\author{
Recommandation du Conseil de l'OCDE, adoptée le 9 mars 1995
}

Les dix questions ci-après au sujet des décisions en matière de réglementation s'inspirent des principes régissant une prise de décision satisfaisante qui sont appliqués dans les pays de l'OCDE en vue d'améliorer l'efficacité et l'efficience de la réglementation officielle en basant celle-ci sur de meilleurs fondements juridiques et factuels, en précisant les options envisageables, en aidant les responsables à prendre de meilleures décisions, en mettant en place des processus de prise de décision clairs et prévisibles, en repérant les réglementations existantes qui sont périmées ou superflues, et en rendant les interventions gouvernementales plus transparentes. Cependant, ces questions doivent s'intégrer dans un système plus large de gestion de la réglementation comportant notamment des éléments comme la collecte et l'analyse d'informations, des processus de consultation et l'évaluation systématique des réglementations existantes.

1. Le problème est-il correctement défini ?

Il conviendrait d'énoncer de façon précise le problème à résoudre en indiquant clairement sa nature et son ampleur, et en expliquant pourquoi il s'est posé (en déterminant les incitations des parties intéressées).

2. L'intervention des pouvoirs publics est-elle justifiée?

L'intervention de l'Etat devrait être fondée sur des éléments indiquant clairement qu'elle est justifiée, vu la nature du problème, les coûts et avantages probables de l'intervention (sur la base d'une évaluation réaliste de l'efficacité des pouvoirs publics) et les autres mécanismes permettant de s'attaquer au problème.

3. La réglementation représente-t-elle la meilleure forme d'intervention gouvernementale ?

Les responsables de la réglementation devraient procéder, à un stade précoce du processus réglementaire, à une comparaison solidement étayée des divers instruments d'action réglementaires et non réglementaires, en examinant des questions importantes comme les coûts, les avantages, les effets sur la répartition des revenus et les nécessités administratives.

4. Quel est le fondement juridique de la réglementation ?

Les processus réglementaires devraient être structurés de manière à garantir que toutes les décisions en matière de réglementation sont rigoureusement conformes aux principes de la légalité, ce qui signifie qu'il faut explicitement veiller a ce que toutes les réglementations soient autorisées par d'autres réglementations fixées à un niveau plus élevé et compatibles avec les obligations découlant de traités et respecter des principes juridiques pertinents tels que la sécurité juridique, la proportionnalité et les formalités prévues.

5. Quel est (quels sont) le(s) niveau(x) d'administration approprié(s) pour agir ?

Les responsables des réglementations devraient choisir le niveau d'administration le plus approprié auquel il convient d'intervenir ou, si plusieurs niveaux sont concernés, mettre au point des systèmes efficaces de coordination entre ces différents niveaux d'administration. 
6. Les avantages des réglementations en justifient-ils les coûts?

Les responsables des réglementations devraient évaluer l'ensemble des coûts et avantages escomptés de chaque réglementation proposée et des options envisageables et communiquer ces estimations sous une forme accessible aux décideurs. Avant de prendre une décision, il conviendrait de s'assurer que les coûts de l'intervention gouvernementale sont justifiés par ses avantages.

7. La répartition des effets au sein de la société est-elle transparente ?

Dans la mesure où l'intervention gouvernementale influe sur la redistribution des revenus et le principe d'équité, les responsables de la réglementation devraient veiller à ce que la répartition des coûts et des avantages des réglementations entre les différents groupes concernés soit transparente.

8. La réglementation est-elle claire, cohérente, compréhensible et accessible aux usagers ?

Les responsables de la réglementation devraient s'assurer que les réglementations seront comprises par les usagers potentiels, et prendre à cette fin les dispositions nécessaires pour clarifier autant que possible le texte et la structure des réglementations.

9. Toutes les parties intéressées ont-elles la possibilité de faire connaître leurs vues ?

Les réglementations devraient être élaborées d'une façon ouverte et transparente, et des procédures appropriées devraient être mises en place pour que les parties intéressées telles que les entreprises et les syndicats concernés, d'autres groupes d'intérêt ou d'autres niveaux d'administration puissent faire part de leurs vues de manière efficace et en temps opportun.

10. Comment le respect de la réglementation sera-t-il assuré ?

Les responsables de la réglementation devraient évaluer les incitations et les institutions par le biais desquelles la réglementation prendra effet, et mettre au point des stratégies d'application efficaces qui permettent d'optimiser leur utilisation. 